\title{
Multimodal News Article Analysis
}

\author{
Arnau Ramisa* \\ Institut de Robòtica i Informàtica Industrial, IRI (UPC-CSIC), Barcelona, Catalonia, Spain \\ Wide Eyes Technologies, Barcelona, Catalonia, Spain \\ aramisa@iri.upc.edu
}

\begin{abstract}
The intersection of Computer Vision and Natural Language Processing has been a hot topic of research in recent years, with results that were unthinkable only a few years ago. In view of this progress, we want to highlight online news articles as a potential next step for this area of research. The rich interrelations of text, tags, images or videos, as well as a vast corpus of general knowledge are an exciting benchmark for high-capacity models such as the deep neural networks. In this paper we present a series of tasks and baseline approaches to leverage corpus such as the BreakingNews dataset.
\end{abstract}

\section{Introduction}

Research at the intersection of traditionally separate artificial intelligence areas, such as Computer Vision and Natural Language Processing, has received a great deal of attention in recent times, with many outstanding results in tasks like automatic image captioning [Vinyals et al., 2015] or image generation from simple sentences [Chang et al., 2014]. These good results in suggest that other, more complex domains, may be ready to be explored.

Nowadays, online news articles have a rich ecosystem of related data coming in many modalities: text, tags, images, captions, videos, comments, mentions on social media, etc. Furthermore, news articles are often interrelated, offering complementary views of the same events. Making sense of this trove of connected data is a very challenging enterprise, both by the scale of the data itself, and by the complexity of the relations at play. Hence, it is a very attractive benchmark on which to test and develop new, powerful artificial intelligence algorithms.

In [Ramisa et al., 2016] the authors present a dataset of a hundred thousand news articles from several high-ranked online newspapers and news portals published during year 2014. In addition to the article text, they include related information, like the pictures accompanying the articles and their captions, geolocation information or reader comments. See

${ }^{*}$ The results presented in this paper are joint work with my coauthors Fei Yan, Francesc Moreno-Noguer and Krystian Mikolajczyk; it is as much theirs as it is mine.

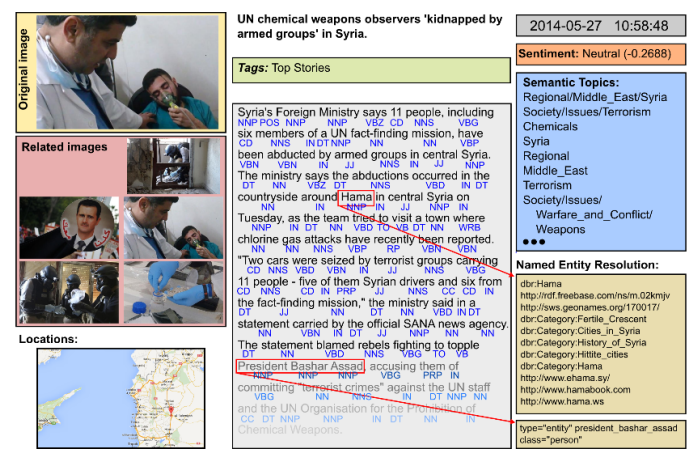

Figure 1: Example article from the BreakingNews dataset.

Figure 1 for statistical information on the dataset. Its size and composition make this dataset an excellent benchmark for data-hungry state-of-the-art deep learning models.

In this paper we will briefly discuss several tasks that can be undertaken using the BreakingNews dataset, plus a baseline approach to tackle some of them.

\section{Tasks Description}

Source detection: This task consists of classifying the newspaper that originally published a given article. One approach to solve it is to model the language and writing preferences, subjects of interest, and sentiment towards certain type of news for each newspaper, but other types of data could be brought into play by also modeling the type of illustrations used, or the number and style of comments posted by the readers (where the sentiment of the audience which the newspaper caters to will be more strongly represented). The task of authorship identification is closely related.

Popularity prediction: The objective of this task is to determine the impact that an article will have after its publication, which can be a useful tool for publishers and community managers. Good indicators of the popularity of an article are the number of times it has been shared on social networks, or the number of comments made by the readers.

Geolocation: The goal in this case is determining the geographical location or locations where the news events take place, expressed as GPS coordinates. Both the Computer Vision [Kalogerakis et al., 2009; Weyand et al., 2016] and the 


\begin{tabular}{|c|c|c|c|c|c|c|c|c|}
\hline Source & $\begin{array}{l}\text { num. } \\
\text { arti- } \\
\text { cles }\end{array}$ & $\begin{array}{l}\text { avg. len. } \\
\text { article }\end{array}$ & $\begin{array}{l}\text { avg. num. } \\
\text { images }\end{array}$ & $\begin{array}{l}\text { avg. len. } \\
\text { caption }\end{array}$ & $\begin{array}{l}\text { avg. num. } \\
\text { comments }\end{array}$ & $\begin{array}{l}\text { avg. len. } \\
\text { com- } \\
\text { ment }\end{array}$ & $\begin{array}{l}\text { avg. num. } \\
\text { shares }\end{array}$ & $\begin{array}{l}\% \text { geo- } \\
\text { located }\end{array}$ \\
\hline Yahoo News & 10,834 & $521 \pm 338$ & $1.00 \pm 0.00$ & $40 \pm 33$ & $126 \pm 658$ & $39 \pm 71$ & $\mathrm{n} / \mathrm{a}$ & $65.2 \%$ \\
\hline BBC News & 17,959 & $380 \pm 240$ & $1.54 \pm 0.82$ & $14 \pm 4$ & $7 \pm 78$ & $48 \pm 21$ & $\mathrm{n} / \mathrm{a}$ & $48.7 \%$ \\
\hline The Irish Independent & 4,073 & $555 \pm 396$ & $1.00 \pm 0.00$ & $14 \pm 14$ & $1 \pm 6$ & $17 \pm 5$ & $4 \pm 20$ & $52.3 \%$ \\
\hline Sydney Morning Herald & 6,025 & $684 \pm 395$ & $1.38 \pm 0.71$ & $14 \pm 10$ & $6 \pm 37$ & $58 \pm 55$ & $718 \pm 4976$ & $60.4 \%$ \\
\hline The Telegraph & 29,757 & $700 \pm 449$ & $1.01 \pm 0.12$ & $16 \pm 8$ & $59 \pm 251$ & $45 \pm 65$ & $355 \pm 2867$ & $59.3 \%$ \\
\hline The Guardian & 20,141 & $786 \pm 527$ & $1.18 \pm 0.59$ & $20 \pm 8$ & $180 \pm 359$ & $53 \pm 64$ & $1509 \pm 7555$ & $61.5 \%$ \\
\hline The Washington Post & 9,839 & $777 \pm 477$ & $1.10 \pm 0.43$ & $25 \pm 17$ & $98 \pm 342$ & $43 \pm 50$ & $\mathrm{n} / \mathrm{a}$ & $61.3 \%$ \\
\hline
\end{tabular}

Table 1: BreakingNews dataset statistics. Mean and standard deviation, usually rounded to the nearest integer.

Natural Language Processing [Serdyukov et al., 2009] communities have approached this problem, and some works have combined both sources of information [Zhou and Luo, 2012] to improve the performance.

Text illustration: In this task the objective is to find the best image to illustrate a news article from a database of images. Variants of the task may include some annotations (e.g. the captions) for the images, or a small gallery of images that can be retrieved together. Many works have addressed this task, some in the news article domain [Feng and Lapata, 2010; 2013], but more commonly in datasets of images with short and accurate descriptions [Barnard et al., 2003; Kovashka et al., 2015; Rasiwasia et al., 2007; Douze et al., 2011]. In the news domain, neither the main body of the article nor the caption of the image offer a faithful description of the picture contents, adding an another layer of difficulty to the problem. Caption generation: A very popular task in recent Computer Vision literature consists on generating short text descriptions of the contents of a picture [Chen and Zitnick, 2015; Donahue et al., 2015; Karpathy and Fei-Fei, 2015; Kiros et al., 2015; Vinyals et al., 2015]. Yet, the task of generating captions for news articles is significantly different since, as mentioned in the previous paragraph, captions are often only indirectly related to the contents of the image. A pioneering example of automatic caption generation for news articles is [Feng and Lapata, 2008].

\section{Text and Image Representation}

In this section we will describe state-of-the-art representations for text and images that can be useful for multimodal news article analysis.

\subsection{Text Representations}

One of the most widely used representations for text documents is the Bag-of-Words (BoW). In this representation, a document is encoded as a histogram with the frequencies with which the words in a vocabulary appear in the text. Despite its simplicity, the Bag-of-Words is a very sound baseline to compare to, as its performance is not far from that of more sophisticated approaches. One caveat of the BoW representation is that very frequent but otherwise non-informative words like "a", "the" or "this" may end up dominating the histogram of counts, thus failing to represent useful information for the subsequent tasks. To counter this undesirable effect often this very common words are excluded from the vocabulary, and ignored when building the histogram. Furthermore, the Term Frequencies (TF) can be weighted by the Inverse Document Frequencies (IDF), which boost the relevance of the most infrequent (and discriminative) words. The BoW with TF-IDF weighting is computed as follows:

$$
D \in \mathbb{R}^{b} \mid D_{j}=t_{j} \log \frac{M}{c^{j}+1}
$$

where $D$ is the $b$-dimensional TF-IDF Bag of Words representation, $M$ is the total number of documents in the corpus, $t_{j}$ is the term frequency of the $j^{\text {th }}$ token (i.e. the number of times it appears in the article), and $c^{j}$ is the document frequency of the token (i.e. the number of training articles where it appears).

In recent years, distributed representations for text, like word2vec, have raised a great deal of attention, due to their excellent performance in many tasks. In contrast with BoW models, these representations do not treat words as atomic units, represented as indices in a vocabulary, but as short, realvalued embeddings based on their context, in a way that preserves semantic similarity. For example, the embedding corresponding to "king" minus that of "man", and plus the one of "woman", will be close to the representation of "queen". These arithmetic properties have been shown to hold across a wide range of linguistic regularities [Mikolov et al., 2013].

In order to scale to large training set sizes (in the billions or even trillions of words) these vector representations are often trained using a simple shallow model and stochastic gradient descent, where the objective to maximize are the log probabilities of words given other words in their context, where the probabilities are estimated using computationally efficient approximations to softmax, like the hierarchical softmax or noise contrastive estimation.

Certain short phrases are not combinations of other words, but have a separate identity. Think for example of the newspaper "Boston Globe": it is not a natural combination of the meanings of "Boston" and "Globe". Consequently, the expressiveness of the model is improved by treating common short phrases as individual tokens.

When it comes to longer sentences or even full documents, however, other strategies have to be considered. One very simple strategy is to do a (weighted) average of all the vector representations in the document, which unfortunately loses the word order information, as in the case of BoW.

Alternatively, in [Le and Mikolov, 2014] the authors propose a learning framework similar to the one for words, where 
an embedding for the words and the documents is optimized simultaneously over the complete corpus. At test time, new documents can be encoded using gradient descent with all the parameters of the model except the document vector held fix. This framework bears some resemblance to the Fisher kernels, a image representation method with successful applications in Computer Vision.

Finally, one can frame the representation of documents as a fixed-size matrix of word embeddings, padded with zeros, and use 1-D temporal convolutions in a Convolutional Neural Network architecture (CNN), popular at the moment thanks to their extraordinary results in Speech Recognition, Computer Vision and other fields [Kim, 2014; Kalchbrenner et al., 2014].

\subsection{Image Representation}

The de facto standard when it comes to image representations in state-of-the-art computer vision literature are the final layers of a deep convolutional neural network trained on the ImageNet dataset [Krizhevsky et al., 2012], or of a fine-tuned version of the network if some training data is available.

Convolutional Neural Networks are a type of feed-forward models that include one or more convolution layers, where responses to patterns in delimited image regions are computed by filters made up of specialized neurons. These filters are applied in each position of a partially overlapped tile over the image, which in practice results in a convolution operation that produces a collection of response maps (one for each filter). The fact that these filters are shared by every position in the tile over the image greatly reduces the number of parameters with respect to fully connected layers, resulting in many advantages, like more efficient architectures, reduced risk of overfitting and a degree of invariance to translations in the input image.

Standard CNN architectures typically project the image through a chain of convolution and pooling layers, after which a last pooling layer, or a number of fully connected layers, reduce the dimensionality further for the classification layer that outputs the predicted class probabilities. These final layers are the ones typically used as a compact representation of the image contents.

As in the case of distributed representations for text, it is possible to average (or max-pool) the vectors of several pictures to obtain a more compact embedding of the whole set.

\section{Learning Models}

In recent literature, Deep Neural Networks models have shown impressive results. Abundant computing power, and large datasets allow these very large models to learn end-toend the best intermediate representation for the objective task, often substantially better than the best hand-crafted representation developed for each particular case.

For multimodal article analysis, we found that the FC7 layer of a standard image classification CNN like VGG19 (after the non-linearity) produced representations of sufficient quality for the article images. Although it would also be feasible to fine-tune the vision $\mathrm{CNN}$ with news article images, for example predicting the tags of the articles given the pictures, in our case this did not yield any significant advantage.

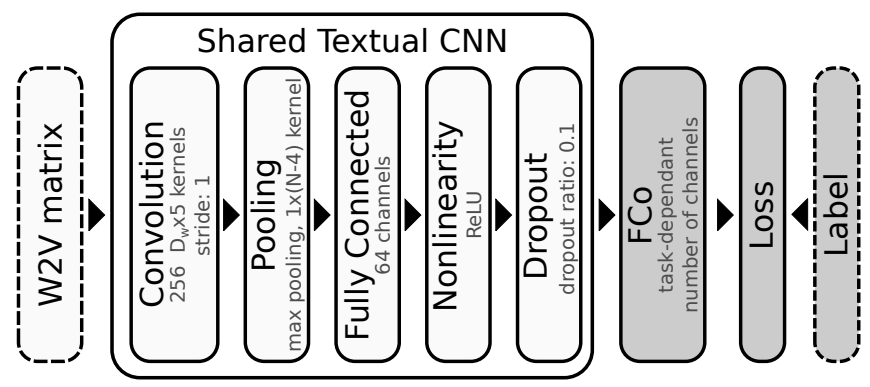

Figure 2: CNN architectures for article analysis, used in the tasks of article illustration, source detection and popularity and geolocation prediction. Dashed boxes are the CNN inputs, and solid boxes correspond to the layers. White layers are shared by all tasks, and shaded layers are task specific.

Regarding the article text representation, we obtained the best results with a small convolutional network that uses the matrices of stacked word $2 \mathrm{vec}$ representations (zero-padded to accommodate to a fixed dimensionality) as input. The network has one convolutional layer and one pooling layer, followed by a fully connected layer, a non-linearity (ReLU proved to be the most numerically stable option) and a dropout layer to regularize the model and prevent overfitting. A graphical representation of the text-processing network can be seen in Figure 2. After this common text processing network, a layer that computes the error between the network predictions and the ground truth labels is necessary to teach the network through backpropagation. Different objectives, or loss layers, can be added to fulfill most of the different tasks in section 2 . Here we will describe some of them.

Multinomial Logistic Loss: This loss maximizes the cross entropy for a one-of-k classification problem. Let $\hat{p}_{i}$ be a predicted probability distribution for the sample $i$ in a batch of $N$ training samples, and $y_{i}$ the ground truth label for the same example; then this loss can be computed for a batch of training examples as:

$$
E=\frac{-1}{N} \sum_{i=1}^{N} \log \hat{p}_{i, y_{i}}
$$

The probability distribution $\hat{p}$ is often obtained using the softmax function on the outputs of the last fully connected layer of a network.

Canonical Correlation Analysis (CCA) loss: The CCA loss is used to find linear projections that maximize the correlation between pairs of corresponding data points in different modalities. Let $Z$ and $Y$ be two $d \times m$ matrices of $m$ aligned data points, then a CCA-based loss can be defined as $L=-\operatorname{corr}(Z, Y)$, whose gradients can be computed using a singular value decomposition of $\Gamma=\Sigma_{z z}^{-1 / 2} \Sigma_{z y} \Sigma_{y y}^{-1 / 2}$, where $\Sigma_{z z}, \Sigma_{y y}$ and $\Sigma_{z y}$ are respectively the covariances of $Z$ and $Y$, and the cross covariances. Please refer to [Ramisa et al., 2016] for a complete derivation of the CCA loss.

L1 distance loss: This loss is simply the L1 distance between the predicted value $z$ and the ground truth label $y$, useful for regression tasks: $L=|z-y|$. 


\begin{tabular}{|c|c|c|c|c|}
\hline \multicolumn{5}{|c|}{ Single task vs Multitask vs Transfer Learning } \\
\hline & & $\begin{array}{c}\text { Geolocation } \\
\text { Median GCD }\end{array}$ & $\begin{array}{l}\text { Popularity } \\
\text { Median } \ell_{1}\end{array}$ & $\begin{array}{c}\text { Source } \\
\text { Bal. acc. }\end{array}$ \\
\hline \multicolumn{2}{|c|}{ Single-task } & 0.90 & 1.09 & 80.7 \\
\hline \multirow{3}{*}{ Multitask } & $\mathrm{G}+\mathrm{P}$ & 1.17 & 1.68 & - \\
\hline & $\mathrm{G}+\mathrm{S}$ & 1.22 & - & 79.1 \\
\hline & $\mathrm{P}+\mathrm{S}$ & - & 1.94 & 79.1 \\
\hline \multirow{6}{*}{ Transfer } & $\mathrm{G} \rightarrow \mathrm{P}$ & - & 1.16 & - \\
\hline & $\mathrm{G} \rightarrow \mathrm{S}$ & - & - & 80.2 \\
\hline & $\mathrm{P} \rightarrow \mathrm{G}$ & 0.97 & - & - \\
\hline & $\mathrm{P} \rightarrow \mathrm{S}$ & - & - & 77.6 \\
\hline & $\mathrm{S} \rightarrow \mathrm{G}$ & 0.92 & - & - \\
\hline & $\mathrm{S} \rightarrow \mathrm{P}$ & - & 0.63 & - \\
\hline
\end{tabular}

Table 2: Comparing single-task, multitask and transfer learing. G: geolocation; P: popularity; S: source. An arrow shows the direction of transfer, for example, $\mathrm{G} \rightarrow \mathrm{P}$ means trained on task $\mathrm{G}$ and transferred to task P.

Great Circle Distance: This loss was introduced in [Ramisa et al., 2016], and computes the geodesic distance between a predicted and a ground truth latitude and longitude pairs. Let the label $\mathbf{y}=\left[y_{1}, y_{2}\right]$, where $y_{1} \in[-\pi / 2, \pi / 2]$ is the latitude and $y_{2} \in[-p i, p i]$ the longitude. Then the output of the network $z \in \mathbb{R}^{2}$ will be the predicted latitude and longitude value, and the Great Circle Distance (GCD) loss can be computed using the spherical law of cosines approximation (and dropping the Earth radius term):

$$
\mathrm{L}=\arccos \left(\sin y_{1} \sin z_{1}+\cos y_{1} \cos z_{1} \cos \delta\right)
$$

where $\delta=z_{2}-y_{2}$. The derivation can be found in [Ramisa et al., 2016].

Furthermore, recent work [Zhang et al., 2014] has shown that sharing a common representation to fulfill several tasks at the same time, can have unforeseen advantageous effects on each of the tasks, as the internal representation of the network can share the "capacities" learned for each one of the tasks to solve the others.

Similarly, networks trained for a particular task can be quickly fine-tuned to solve another related objective with very little computational effort and training data, sometimes even surpassing the performance obtained by a network directly trained on the target task.

\section{Results and Conclusions}

In this section we present the particular experimental details and results obtained with the BreakingNews dataset.

We split the dataset according to the standard partitions ${ }^{1}$, which gives training, validation and tests sets of $60 \%, 20 \%$ and $20 \%$ respectively. The textual representations used are a TF-IDF BoW with a vocabulary of 44,665 dimensions, and a word2vec embedding of 500 dimensions computed on the training set. The images are represented as concatenated

\footnotetext{
${ }^{1}$ http://www.iri.upc.edu/people/aramisa/ BreakingNews/index.html
}

ReLU7 features from the ImageNet VGG19 and PLACES CNNs, and the model architectureis that of Figure 2 with the appropriate loss function(s).

For article illustration we measure performance primarily using the median rank (lower is better). For each test article we rank the 23,200 test images according to the projection learned by the CNN or by a CCA model. The best results are very similar with both methods: 405 for the CNN model using a W2V matrix and the concatenation of PLACES and VGG19 RelU7 features, and 397 for the CCA using the same visual representation and the BoW TF-IDF for the text.

The dataset includes a set of five related images for each article, that often include the actual article image. If we construct the image representation as the average of their VGG19 ReLU7 vectors, median rank goes down to 137 for CCA, which is a significant improvement. If we add the caption information to the representation on the image side, the median rank drops to 7 , and if we anonimize the captions by replacing person names, locations and organizations to make them more neutral, it is still reduced by an order of magnitude to 42 .

The remaining three tasks are evaluated individually, and in a multitask and transfer learning scenarios (see Table 2). For multitask, we jointly train a model with each of the three pairs of tasks. For transfer learning, we train six models using the three pairs and in both directions.

Geolocation is evaluated in terms of median Great Circle Distance between the predicted and the ground truth locations, popularity on the median $l_{1}$ distance between the predicted and real number of comments, and source prediction based on the balanced classification accuracy. The results in Table 2 show that single-task learning tends to have the best performance, and that transfer learning in general outperforms multitask learning. The fact that multitask learning does not perform as well as the other alternatives seems to indicate that forcing the lower layers of the $\mathrm{CNN}$ to share common representations harms the performance of both tasks, and that our tasks are not as "compatible" as those considered in [Zhang et al., 2014]. CNN architectures that further decouple the tasks did not improve the results.

On the other hand, in most cases the performance of transfer learning is comparable to that of single-task learning. When transferring from "source" to "popularity" prediction, the performance is even higher than single-task "popularity" prediction. Indicating that the low and mid-level representations learned by source prediction helped the other task. For multitask and transfer learning, we set the learning rate to one tenth of the base learning rate, to help the layers change slowly and reduce the co-adaptation effect [Yosinski et al., 2014].

\section{Acknowledgements}

This work was partly funded by the ERA-net CHISTERA project VISEN PCIN-2013-047, the MINECO project RobInstruct TIN2014-58178-R, and project EPSRC UK EP/N007743/1. The authors would like to thank NVidia for the hardware donation under the GPU grant program. 


\section{References}

[Barnard et al., 2003] Kobus Barnard, Pinar Duygulu, David Forsyth, Nando De Freitas, David Blei, and Michael Jordan. Matching words and pictures. The Journal of Machine Learning Research, 3:1107-1135, 2003.

[Chang et al., 2014] Angel Chang, Manolis Savva, and Christopher Manning. Interactive learning of spatial knowledge for text to $3 \mathrm{~d}$ scene generation. Sponsor: Idibon, page 14, 2014.

[Chen and Zitnick, 2015] Xinlei Chen and C. Lawrence Zitnick. Mind's eye: A recurrent visual representation for image caption generation. In CVPR, 2015.

[Donahue et al., 2015] Jeff Donahue, Lisa Anne Hendricks, Sergio Guadarrama, Marcus Rohrbach, Subhashini Venugopalan, Kate Saenko, and Trevor Darrell. Long-term recurrent convolutional networks for visual recognition and description. In $C V P R, 2015$.

[Douze et al., 2011] Matthijs Douze, Arnau Ramisa, and Cordelia Schmid. Combining attributes and fisher vectors for efficient image retrieval. In CVPR, pages 745-752, 2011.

[Feng and Lapata, 2008] Yansong Feng and Mirella Lapata. Automatic image annotation using auxiliary text information. In Proceedings of ACL-08: HLT, pages 272-280, Columbus, Ohio, 2008.

[Feng and Lapata, 2010] Yansong Feng and Mirella Lapata. Topic Models for Image Annotation and Text Illustration. Conference of the North American Chapter of the ACL: Human Language Technologies, (June):831-839, 2010.

[Feng and Lapata, 2013] Yansong Feng and Mirella Lapata. Automatic caption generation for news images. IEEE Transactions on Pattern Analysis and Machine Intelligence, 35(4):797-812, 2013.

[Kalchbrenner et al., 2014] Nal Kalchbrenner, Edward Grefenstette, and Phil Blunsom. A convolutional neural network for modelling sentences. In ACL, 2014.

[Kalogerakis et al., 2009] Evangelos Kalogerakis, , Olga Vesselova, James Hays, Alexia Efros, and Aaron Hertzmann. Image sequence geolocation with human travel priors. In ICCV, pages 253-260, 2009.

[Karpathy and Fei-Fei, 2015] Andrej Karpathy and Li FeiFei. Deep visual-semantic alignments for generating image descriptions. In CVPR, 2015.

[Kim, 2014] Yoon Kim. Convolutional neural networks for sentence classification. In EMNLP, 2014.

[Kiros et al., 2015] Ryan Kiros, Ruslan Salakhutdinov, and Richard Zemel. Unifying visual-semantic embeddings with multimodal neural language models. TACL, 2015.

[Kovashka et al., 2015] Adriana Kovashka, Devi Parikh, and Kristen Grauman. Whittlesearch: Interactive image search with relative attribute feedback. International Journal of Computer Vision, pages 1-26, 2015.
[Krizhevsky et al., 2012] Alex Krizhevsky, Ilya Sutskever, and Geoffrey Hinton. ImageNet classification with deep convolutional neural networks. In NIPS, 2012.

[Le and Mikolov, 2014] Quoc Le and Tomas Mikolov. Distributed representations of sentences and documents. In ICML, 2014.

[Mikolov et al., 2013] Tomas Mikolov, Ilya Sutskever, Kai Chen, Greg Corrado, and Jeffrey Dean. Distributed representations of words and phrases and their compositionality. In NIPS, 2013.

[Ramisa et al., 2016] Arnau Ramisa, Fei Yan, Francesc Moreno-Noguer, and Krystian Mikolajczyk. Breakingnews: Article annotation by image and text processing. arXiv:1603.07141 [cs.CV], 2016.

[Rasiwasia et al., 2007] Nikhil Rasiwasia, Pedro Moreno, and Nuno Vasconcelos. Bridging the gap: Query by semantic example. IEEE Transactions on Multimedia, 9(5):923-938, 2007.

[Serdyukov et al., 2009] Pavel Serdyukov, Vanessa Murdock, and Roelof Van Zwol. Placing flickr photos on a map. In ACM Conference on Research and Development in Information Retrieval, pages 484-491. ACM, 2009.

[Vinyals et al., 2015] Oriol Vinyals, Alexander Toshev, Samy Bengio, and Dumitru Erhan. Show and tell: A neural image caption generator. In CVPR, pages 3156-3164, 2015.

[Weyand et al., 2016] Tobias Weyand, Ilya Kostrikov, and James Philbin. Planet - photo geolocation with convolutional neural networks. arXiv:1602.05314 [cs.CV], 2016.

[Yosinski et al., 2014] Jason Yosinski, Jeff Clune, Yoshua Bengio, and Hod Lipson. How transferable are features in deep neural networks? In NIPS, pages 3320-3328, 2014.

[Zhang et al., 2014] Zhanpeng Zhang, Ping Luo, Chen Loy, and Xiaoou Tang. Facial landmark detection by deep multitask learning. In ECCV, 2014.

[Zhou and Luo, 2012] Youjie Zhou and Jiebo Luo. Geolocation inference on news articles via multimodal pLSA. ACM International Conference on Multimedia, page 741, 2012. 